\title{
Translational energy dependence of reaction mechanism: $\mathrm{Xe}^{+}+\mathrm{CH}_{4} \rightarrow \mathrm{XeH}^{+}+\mathrm{CH}_{3}$
}

\author{
G. D. Miller, ${ }^{\text {a) }}$ L. W. Strattan, ${ }^{\text {b) }}$ C. L. Cole, and P. M. Hierl \\ Department of Chemistry, University of Kansas, Lawrence, Kansas 66045 \\ (Received 21 December 1979; accepted 4 April 1980)

\begin{abstract}
The dynamics of the exoergic ion-molecule reaction $\mathrm{Xe}^{+}\left(\mathrm{CH}_{4}, \mathrm{CH}_{3}\right) \mathrm{XeH}^{+}$were studied by chemical accelerator techniques over the relative translational energy range 0.2 to $8 \mathrm{eV}$. Results of the kinematic measurements are reported as scattering intensity contour maps in Cartesian velocity space. Center-ofmass angular and energy distributions, derived from these maps, provide information on the reaction mechanism and on the partitioning of available energy between internal and translational modes in the products. The results suggest that reaction proceeds via the formation of a long-lived complex at low collision energies (below $0.5 \mathrm{eV}$ ) and by a direct mechanism approaching spectator stripping at higher energies.
\end{abstract}

\section{INTRODUCTION}

Recently we have reported kinematic data and excitation functions (integral reaction cross sections versus collision energy) for ion-molecule reactions of the type

$$
\mathrm{X}^{+}+\mathrm{CH}_{4}-\mathrm{XH}^{+}+\mathrm{CH}_{3} \text {, }
$$

where $\mathrm{X}=\mathrm{Ar},{ }^{1,2} \mathrm{Kr},{ }^{3,4}$ and $\mathrm{N}_{2} \cdot{ }^{5}$ In this paper, we report the results of an extension of these studies to the case where $\mathrm{X}=\mathrm{Xe}$.

In the previous studies Reaction (1) was found to be dominated by a direct mechanism (i.e., to proceed on a time scale shorte $r$ than a typical rotational period) over the entire energy range studied (approximately 0.4 to 35 $\mathrm{eV}$ in the center-of-mass system). Although energy partitioning in these reactions could be roughly described by the spectator stripping model (SSM), this simple model for direct reaction consistently overestimated the product translational energy by about $0.1 \mathrm{eV}$. The excitation functions for these strongly exoergic reactions were found to maximize at about $5 \mathrm{eV}$ (c.m.) and then to decrease sharply at lower collision energies, appearing to possess a translational energy threshold at about 0.1 eV. The negative deviations from the SSM and the apparent translational energy thresholds led us to suggest that the potential energy hypersurface for each of these reactions contains a basin which impedes product separation and which, at the lowest collision energies, could result in the formation of an $\mathrm{XCH}_{4}^{+}$complex which preferentially decomposes via various exothermic charge exchange channels rather than by $\mathrm{XH}^{+}$formation. ${ }^{6}$

$\mathrm{Xe}^{+}$, however, differs from the ions previously studied in that charge transfer between methane and the ground state of the reactant ion is endothermic and is, therefore, energetically forbidden at low collision energies. Consequently, it is anticipated that the present study might provide additional information on complex formation and the subsequent competition between charge transfer and chemical reaction (i.e., atomic rearrangement) at low collision energies.

\footnotetext{
a) Present address: Monsanto Mound Laboratory, P. O. Box 32, Miamisburg, Ohio 45342 .

b) Present address: Environmental Protection Agency, Surveillance and Analysis Division, Kansas City, Kansas 66115.
}

Ion-molecule reactions (including charge transfer) in xenon-methane mixtures have previously been studied by high-pressure mass spectrometry, ${ }^{7}$ ion cyclotron resonance, ${ }^{8,9}$ and tandem mass spectrometry. ${ }^{10,11}$

\section{EXPERIMENTAL}

\section{A. Apparatus and method}

The instrument used in this study is a single beamcollision chamber type of chemical accelerator with which product velocity and angular analyses are possible. $\mathrm{Xe}^{+}$ions, formed by impact of $33 \mathrm{eV}$ electrons, are focused into a nearly monoenergetic beam of variable energy ( 1 to $100 \mathrm{eV}$ laboratory) by an ion lens system. ${ }^{12}$ This collimated beam [with a typical angular spread of $1^{\circ}$ full width at half-maximum (FWHM)] passes through the collision chamber containing methane at a pressure of about $10^{-3}$ Torr (measured by a capacitance manometer) and a temperature of $358^{\circ} \mathrm{K}$ (i.e., under single collision conditions). The ion gun can be rotated about the center of the collision chamber, thus permitting the fixed detector to measure the scattered ionic products at various laboratory angles, $\theta$. Those ions leaving the collision chamber at the selected angle pass through a rectangular detection slit [typical resolution is $1^{\circ}$ in the horizontal $(\theta)$ plane and $4^{\circ}$ in the vertical $(\Phi)$ plane], a parallel plate retarding potential energy analyzer, and a set of strong focussing quadrupole lenses. ${ }^{13}$ Mass analysis of the ions is performed with the $30 \mathrm{~cm}, 90^{\circ} \mathrm{de}-$ flection magnetic sector analyzer of a Nuclide mass spectrometer from which the conventional ion source and accelerating electrodes have been removed. The individual components of this instrument have been described in detail elsewhere. ${ }^{14}$

Because the primary ion beam was not mass selected, it contained a mixture of xenon isotopes whose relative intensities are determined by their natural abundances. The ${ }^{132} \mathrm{Xe}$ isotope was utilized for these studies because it is the most abundant isotope (27\%) and because there is no mass $133 \mathrm{amu}$ isotope of Xe to interfere with the analysis of the ${ }^{192} \mathrm{XeH}^{*}$ product.

\section{B. Internal reactant states}

Because the $\mathrm{Xe}^{+}$is produced by impact of $33 \mathrm{eV}$ electrons, the primary ion beam contains no doubly charged 
ions and no more than $2 \%$ high-energy metastable ions. ${ }^{15}$ Nearly all $(98 \%)$ of the $\mathrm{Xe}^{+}$ions, therefore, are in the ${ }^{2} P$ state and are presumably distributed statistically in a $2: 1$ ratio between the ${ }^{2} P_{3 / 2}$ ground state and the ${ }^{2} P_{1 / 2}$ excited state, which differ in energy by $1.31 \mathrm{eV} .^{18}$

The neutral target gas is in thermal equilibrium, so that its internal energy is described by the Boltzmann distribution function. At $358^{\circ} \mathrm{K}$, nearly all of the methane molecules are in their ground vibrational state and the most probable rotational state is $J=9$, which corresponds to $0.015 \mathrm{eV}$ of rotational energy. Consequently, nearly all of the energy available to the reaction products comes from reactant translation and the heat of reaction.

\section{Heats of reaction}

Since the ionization potential of $\mathrm{CH}_{4}$ is $12.6 \mathrm{eV},{ }^{17}$ while that of $\mathrm{Xe}$ is $12.130 \mathrm{eV}$ for formation of $\mathrm{Xe}^{+}\left({ }^{2} P_{3 / 2}\right)$ and $13.436 \mathrm{eV}$ for formation of $\mathrm{Xe}^{+}\left({ }^{2} P_{1 / 2}\right)$, charge trans fer to $\mathrm{CH}_{4}$ is endothermic by $0.47 \mathrm{from}$ the ground state of $\mathrm{Xe}^{+}$but exothermic by 0.84 from the excited ${ }^{2} P_{1 / 2}$ state.

The energetics of the $\mathrm{H}$-atom abstraction reaction

$$
\mathrm{Xe}^{+}\left({ }^{2} P_{J}\right)+\mathrm{CH}_{4}\left({ }^{1} A_{1}\right) \rightarrow \mathrm{XeH}^{+}\left(X^{1} \Sigma^{+}\right)+\mathrm{CH}_{3}\left({ }^{2} A_{2}^{\prime \prime}\right)
$$

are somewhat less certain. Flowing afterglow measurements of equilibrium in $\mathrm{Xe}-\mathrm{CO}_{2}-\mathrm{H}_{2}$ mixtures ${ }^{18}$ indicate that the proton affinity (PA) of Xe is $0.42 \pm 0.07 \mathrm{eV}$ less than that of $\mathrm{CO}_{2}$. With the recently reported value of $\mathrm{PA}\left(\mathrm{CO}_{2}\right)=5.51 \pm 0.04 \mathrm{eV},{ }^{19}$ this gives $\mathrm{PA}(\mathrm{Xe})=5.09$ $\pm 0.11 \mathrm{eV}$. Assuming a value of $4.477 \mathrm{eV}$ for $D_{0}\left(\mathrm{H}-\mathrm{CH}_{3}\right),{ }^{17}$ one finds that Reaction (2) is endothermic by $0.86 \pm 0.11 \mathrm{eV}$ for the $J=\frac{3}{2}$ state of $\mathrm{Xe}^{+}$but exothermic by $0.45 \pm 0.11 \mathrm{eV}$ for the $J=\frac{1}{2}$ state.

On the other hand, theoretical calculations of the $\mathrm{XeH}^{*}$ potential $^{20}$ which agree well with experimental data ${ }^{21}$ on the elastic scattering of protons by Xe yield a well depth of $5.81 \mathrm{eV}$ for $\mathrm{H}^{+}-\mathrm{Xe}$. If one assumes that the zero-point vibrational energy of $\mathrm{XeH}^{+}$is $0.14 \mathrm{eV}$ (equal to that of the isoelectronic molecule $\mathrm{HI}$ ), ${ }^{22}$ one obtains $\mathrm{PA}(\mathrm{Xe})=5.67 \mathrm{eV}$. With this value, Reaction (2) would be endothermic by $0.28 \mathrm{eV}$ for the ground state of $\mathrm{Xe}^{+}$and exothermic by $1.03 \mathrm{eV}$ for the excited state of the reactant ion.

Moreover, in an early high-pressure mass spectrometric study of Reaction (2), Franklin and Field ${ }^{7}$ found that the appearance potential of $\mathrm{XeH}^{+}$was equal (within experimental error) to that of $\mathrm{Xe}^{+}$. This suggests that $\mathrm{H}$-atom abstraction is exothermic even with ground state $\mathrm{Xe}^{+}\left({ }^{2} P_{3 / 2}\right)$ ions.

\section{RESULTS}

\section{A. Excitation functions}

Integral reaction cross sections $\sigma$ are calculated from the formula ${ }^{2}$

$$
\sigma=\frac{I_{c} / I_{A}}{n_{B} L} K(T),
$$

where $I_{C}$ is the total reactively scattered product $\left({ }^{132} \mathrm{XeH}^{+}\right)$ion intensity, $I_{A}$ is the primary $\left({ }^{132} \mathrm{Xe}^{+}\right)$ion intensity, $n_{B}$ is the number density of $\mathrm{CH}_{4}$ in the collision
TABLE I. Integral cross sections for the reaction $\mathrm{Xe}^{+}+\mathrm{CH}_{4}$

\begin{tabular}{|c|c|c|c|}
\hline $\begin{array}{l}\text { Initial } \\
\text { translational } \\
\text { energy, } \\
T(\mathrm{e} V)\end{array}$ & $\begin{array}{l}\text { Relative } \\
\text { collection } \\
\text { efficiency, } \\
K_{C} / K_{A}{ }^{a}\end{array}$ & $\begin{array}{l}\text { Transmission } \\
\text { factor, } K(T)^{\mathrm{b}}\end{array}$ & $\begin{array}{l}\text { Integral reaction } \\
\text { cross sections, } \\
\sigma\left(10^{-6} \mathrm{~cm}^{2}\right)^{\mathrm{c}}\end{array}$ \\
\hline 1.56 & 2.98 & 0.54 & 0.54 \\
\hline 1.57 & 2.24 & 0.71 & 0.69 \\
\hline 1.61 & 1.55 & 0.79 & 0.47 \\
\hline 1.84 & 1.43 & 0.78 & 0.55 \\
\hline 2.15 & 1.69 & 0.88 & 0.84 \\
\hline 2.20 & 2.04 & 0.70 & 0.48 \\
\hline 2.36 & 4.82 & 0.79 & 0.43 \\
\hline 2.48 & 1.03 & 0.81 & 0.40 \\
\hline 2.80 & 1.16 & 0.80 & 0.47 \\
\hline 2.96 & 2.27 & 0.84 & 0.36 \\
\hline 3.38 & 1.21 & 0.79 & 0.47 \\
\hline 3.70 & 1.90 & 0.84 & 0.48 \\
\hline 4.38 & 1.25 & 0.85 & 0.41 \\
\hline 5.18 & 1.22 & 0.83 & 0.45 \\
\hline 7.63 & 1.12 & 0.82 & 0.14 \\
\hline
\end{tabular}
$\rightarrow \mathrm{XeH}^{+}+\mathrm{CH}_{3}$.

${ }^{a}$ Ratio of collection efficiency correction factors (see Sec. III A).

${ }^{\mathrm{b}}$ Correction factor for attenuation of primary beam, calculated from Eq. (4).

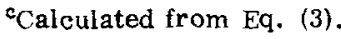

chamber, and $L$ is the collision path length. The familiar thin target formula, $I_{C} / I_{A} n_{B} L$, is corrected for any attenuation of the reactant and product beams by the factor ${ }^{2}$

$$
K(T)=T \ln T /(T-1),
$$

where $T$, the transmission, is the ratio of transmitted to incident primary beam intensities, $I_{A} / I_{A}^{\circ}$. As before the ratio of total intensities is given by

$$
\frac{I_{c}}{I_{A}}=\frac{I_{C}\left(0^{\circ}\right) K_{C}}{I_{A}\left(0^{\circ}\right) K_{A}} K_{1} K_{2}
$$

where $I_{A}\left(0^{\circ}\right)$ and $L_{C}\left(0^{\circ}\right)$ are the observed ion intensities at the maxima in the laboratory angular distributions of $A$ and $C, K_{A}$, and $K_{C}$ correct for differences in the collection efficiencies due to differences in the angular distributions of reactants and products, $K_{1}$ corrects for differences in multiplier gain, and $K_{2}$ corrects for broadening of the mass spectral peak when ions are formed with a wide distribution of translational energies.

Because the small cross section for Reaction (2) resulted in very low product ion intensities, the quantities $K_{1}$ and $K_{2}$ could not be measured directly and were therefore assumed to equal unity. This assumption is consis tent with results obtained in previous studies ${ }^{1,2,4}$ of $\mathrm{sim}$ ilar reactions and is not expected to introduce an error of more than a few percent. The correction factor $K_{C} /$ $K_{A}$ was obtained directly by appropriate integration of the experimentally measured laboratory angular distributions. Table I presents the integral cross sections calculated from Eq. (3), along with the experimentally determined quantities $K_{C} / K_{A}$ and $K(T)$. The uncertainties in the cross sections are estimated to be $\pm 40 \%$ relative to each other and $\pm 60 \%$ on an absolute scale. The excitation function ( $\sigma$ versus $T$ ) for Reaction (2) is presented in Fig. 1. 


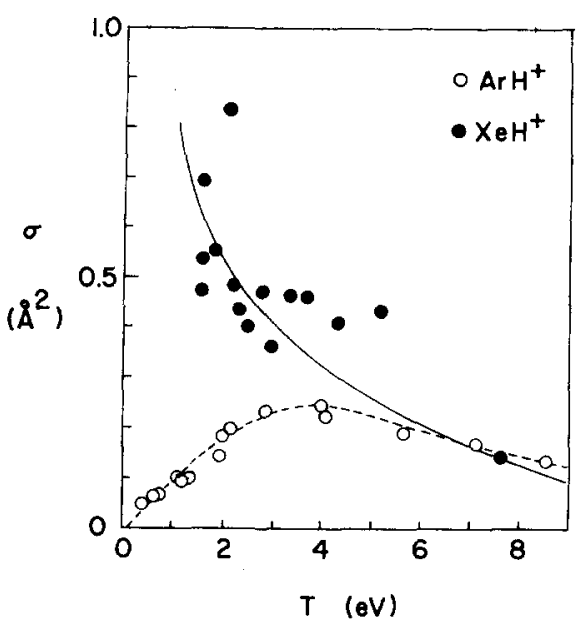

FIG. 1. Integral reaction cross section $\sigma$ vs initial relative translational energy $T$ for the reaction $\mathrm{Xe}^{+}+\mathrm{CH}_{4} \rightarrow \mathrm{XeH}^{+}+\mathrm{CH}_{3}$ (solid circles). The values of the cross sections, calculated from Eq. (3), are listed in Table I. The solid line represents an empirical fit to the experimental data. Also shown (open circles) are cross sections taken from Ref. 2 for the reaction $\mathrm{Ar}^{+}+\mathrm{CH}_{4} \rightarrow \mathrm{ArH}^{+}+\mathrm{CH}_{3}$.

\section{B. Kinematic data}

Angular $(\theta)$ distributions in the laboratory reference frame are obtained for $\mathrm{Xe}^{+}$and $\mathrm{XeH}^{+}$by recording the appropriate ion signal while rotating the ion gun about the center of the collision chamber. Because the detector views a decreasing fraction of the collision path length with increasing scattering angle, the observed ion signal at each laboratory angle is divided by the path length subtended by the detector at that angle. ${ }^{2}$ The resulting angular distributions, $I_{L}\left(\theta, \Phi=0^{\circ}\right)$, represent the relative ion intensity scattered through a laboratory angle $\theta$ in the plane $\Phi=0^{\circ}$ from a reaction path of unit length.

The stopping potential curves obtained by energy analysis at various laboratory angles are first scaled to reflect the total relative intensity at that angle and are

$$
\begin{aligned}
& \mathrm{Xe}^{+}+\mathrm{CH}_{4} \rightarrow \mathrm{XeH}^{+}+\mathrm{CH}_{3} \\
& \mathrm{~T}=7.31 \mathrm{eV}
\end{aligned}
$$
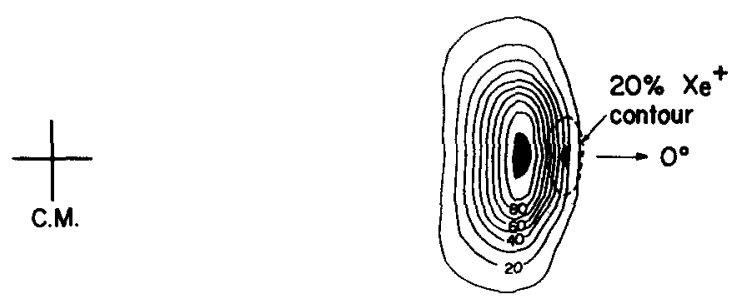

$$
\widetilde{200 \mathrm{~m} / \mathrm{s}}-20^{\circ}
$$

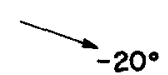

FIG. 2. Product probability distribution for Reaction (2) at the relative collision energy $T=7.31 \mathrm{eV}$. The product ion intensities, normalized to 100 at the position of maximum intensity, are shown relative to the Cartesian system $P_{C}$. Arrows represent scattering angle with respect to the center of mass (marked C.M.). The dashed oval represents the $20 \%$ contour line for the reactant $\mathrm{Xe}^{*}$.

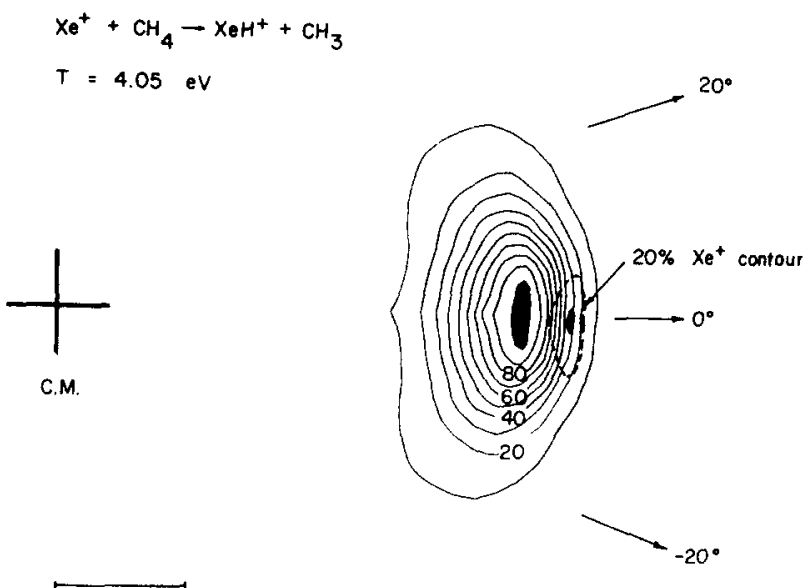

$200 \mathrm{~m} / \mathrm{sec}$

FIG. 3. Product probability distribution for Reaction (2) at $T=4.05 \mathrm{eV}$.

then differentiated to yield energy distributions at that angle, $I_{L}\left(E, \theta, \Phi=0^{\circ}\right)$. These energy distributions are converted to velocity distributions by multiplying each point by the corresponding laboratory velocity $v$ in accord with the transformation ${ }^{23}$

$$
I_{L}(E, \Theta, \Phi) d E d \Omega=I_{L}(v, \Theta, \Phi) d v d \Omega .
$$

These laboratory cross sections, $I_{L}(v, \Theta, \Phi)$, are converted to probabilities in Cartesian velocity space according to the transformation

$$
P_{C}\left(v_{x}, v_{y}, v_{z}\right)=v^{-2} I_{L}(v, \Theta, \Phi)
$$

where $P_{C}$ represents the probability of finding product in a given volume of velocity space. ${ }^{23}$ These probabilities are then scaled, with the highest intensity arbitrarily set equal to 100 . A plot of the appropriate contours on a velocity vector diagram produces a map of relative intensities as seen by a detector sensitive to particles in an element $d v_{x} d v_{y} d v_{z}$ of velocity space. Such velocity vector diagrams have been constructed to present Cartesian probabilities for the formation of $\mathrm{XeH}^{+}$(Figs. $2-7$ ) at various relative collision energies over the range 0.43 to $7.3 \mathrm{eV}$.

Center-of-mass angular distributions, $I(\theta)$, for reac-

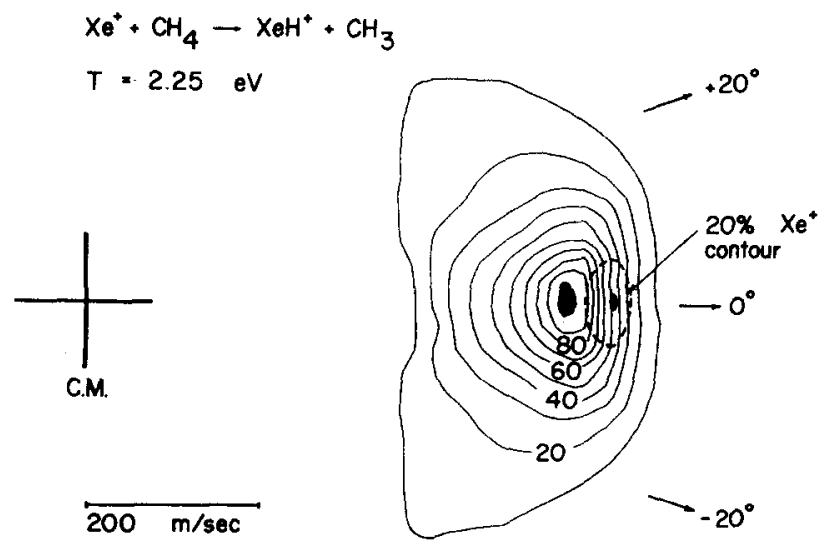

FIG. 4. Product probability distribution for Reaction (2) at $T=2.25 \mathrm{eV}$. 


$$
\begin{aligned}
& \mathrm{Xe}^{+}+\mathrm{CH}_{4}-\mathrm{XeH}^{+}+\mathrm{CH}_{3} \\
& T=1.58 \mathrm{eV}
\end{aligned}
$$

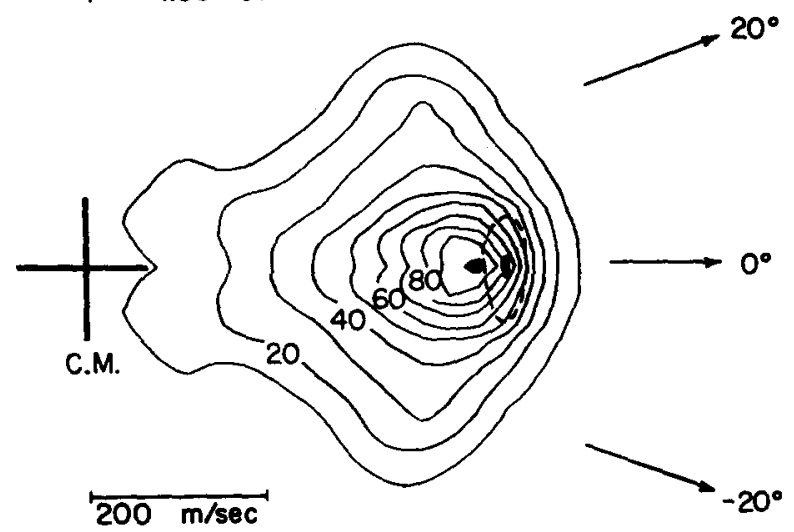

FIG. 5. Product probability distribution for Reaction (2) at $T=1.58 \mathrm{eV}$.

tively scattered products are derived from the velocity vector diagrams using the relation

$$
I(\theta)=\int_{0}^{\infty} u^{2} P_{C}(u, \theta) d u,
$$

where $P_{C}(u, \theta)$ is the Cartesian probability of finding product at the c.m. velocity $u$ and scattering angle $\theta$. The results, presented as differential polar cross section, $d \sigma / d=2 \pi I(\theta) \sin \theta$, are shown in Figs. 10 and 11 . It is possible to obtain absolute values for these differential cross sections by using the measured values of the integral cross sections and the relation

$$
\sigma=2 \pi \int_{0}^{\pi} I(\theta) \sin \theta d \theta
$$

Product relative translational energy distributions $P\left(T^{\prime \prime}\right)$, are derived from the velocity vector diagrams using the relation

$$
\begin{aligned}
P\left(T^{\prime}\right)= & 2 \pi \int_{0}^{\pi} u^{2} P_{C}(u, \theta) \sin \theta d \theta \frac{d u}{d T^{\prime}} \\
& \propto 2 \pi \int_{0}^{\pi} u P_{C}(u, \theta) \sin \theta d \theta .
\end{aligned}
$$

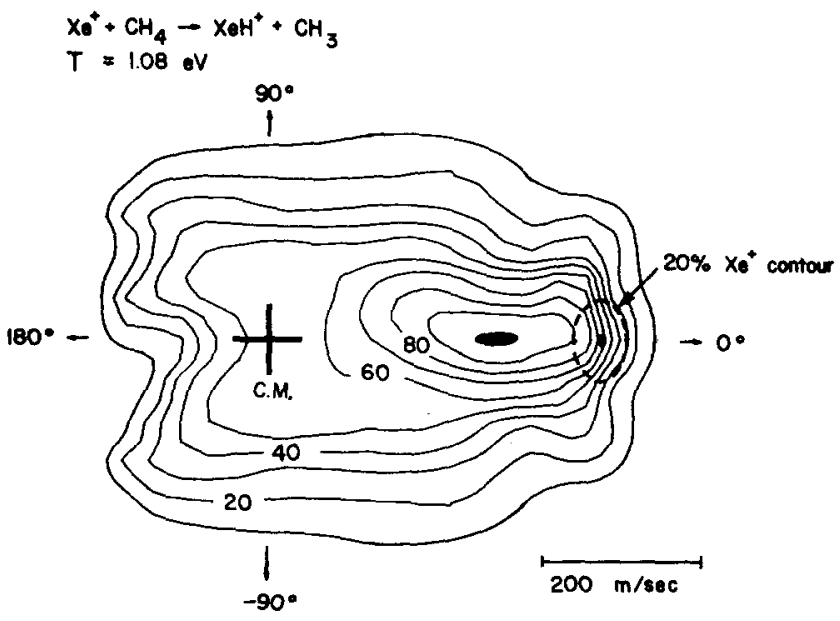

FIG. 6. Product probability distribution for Reaction (2) at $T=1.08 \mathrm{eV}$.

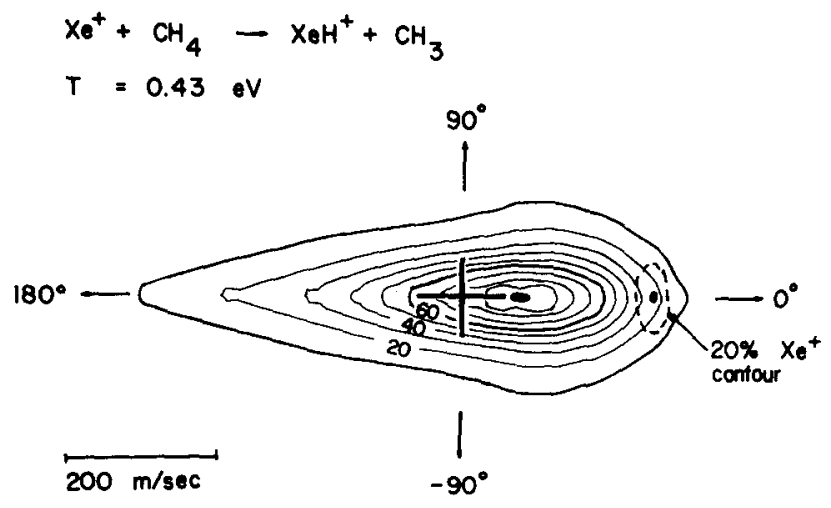

FIG. 7. Product probability distribution for Reaction (2) at $T=0.43 \mathrm{eV}$.

These distributions are then used to calculate the probability, $P(Q)$, that the relative translational energy of the system changes by an amount $Q=T^{\prime}-T$. The results, obtained at six collision energies over the range $0.43-7.31 \mathrm{eV}$ (c.m.), are shown in Fig. 12 where $P(Q)$ is plotted versus the translational energy loss, $-Q$.

As an alternative to the construction of a complete contour map and subsequent de rivation of complete product relative translational energy distributions using Eq. (10), the position of maximum product ion intensity was simply determined in a number of experiments by the method of measuring the product energy distribution at the laboratory angle of maximum product intensity and then converting this distribution to the corresponding Cartesian velocity spectrum by multiplying the intensity at each point (i.e., energy) by the overall Jacobian factor of $1 / v$. The velocity corresponding to the peak in this velocity spectrum was then used to calculate the most probable value of the translational exoergicity, $Q_{m p}$. The values so obtained, as well as those derived from the data shown in Fig. 12, are plotted versus the relative collision energy in $\mathrm{Fig} .13$.

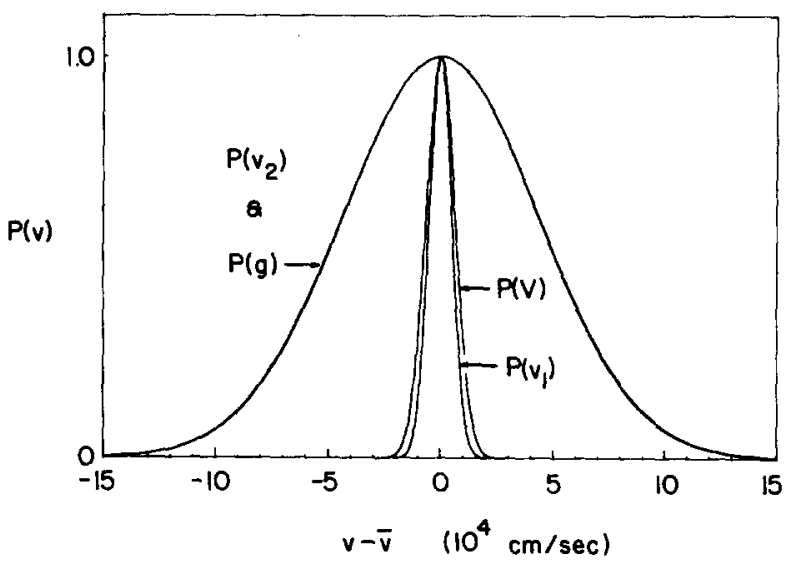

FIG. 8. Calculated distribution functions for the projectile velocity $\left(v_{1}\right)$, the target velocity $\left(v_{2}\right)$, the relative velocity $(g)$, and the velocity of c.m. of the system $(V) .\left[P(g)\right.$ and $P\left(v_{2}\right)$ are so similar that they appear as a single curve in this figure.] These functions were calculated from Eqs. (A4), (A3), (A5), and $(\mathrm{A} 8)$, respectively, using the parameters listed in Table II. 


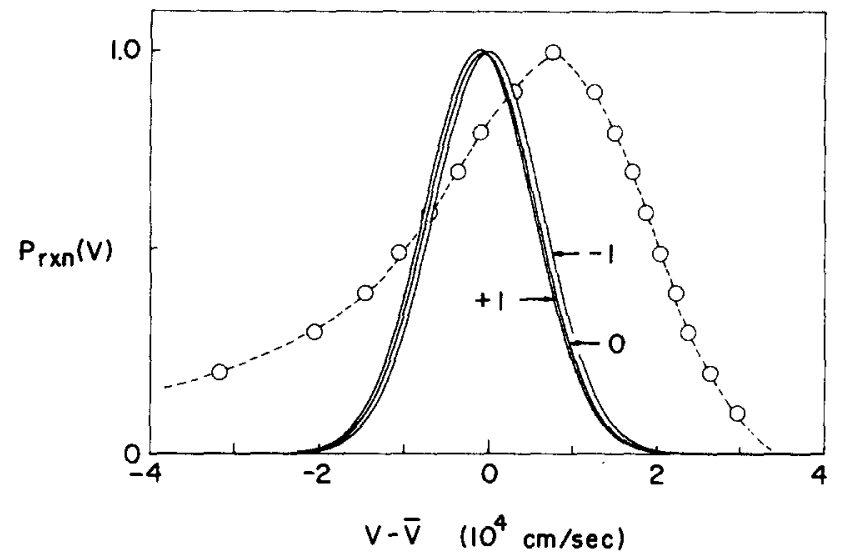

FIG. 9. Solid lines show $P_{r+n}(V)$, the distribution of centroids for reactive collisions in which the reaction cross section varies with relative velocity $g$ as $\sigma(g) \propto g^{s}$. For the three cases, $s=+1,0,-1$, the function $P_{r \times n}(V)$ was calculated from Eqs. (A11) and (A17)-(A19), using the parameters listed in Table II. The open circles represent the measured velocity distribution along the $0^{\circ}$ axis for the $\mathrm{XeH}^{+}$product in the experiment at $0.43 \mathrm{eV}$ relative collision energy.

\section{DISCUSSION}

\section{A. Excitation function}

The integral reaction cross sections for production of $\mathrm{XeH}^{*}$ shown in Fig. 1 vary from about 1.0 to $0.1 \AA^{2}$ over the range of collision energies from 1.5 to $8 \mathrm{eV}$. It should be noted, however, that these values for the cross section are based upon measurement of the entire primary $\mathrm{Xe}^{+}$ion current and thus are averages of the actual cross sections for the two states of $\mathrm{Xe}^{+}$present, weighted according to their relative abundances in the primary beam. As discussed in Sec. IIC, Reaction (2) is endothermic for $\mathrm{Xe}^{+}$ions in the ${ }^{2} P_{3 / 2}$ ground state but exothermic for $\mathrm{Xe}^{+}\left({ }^{2} P_{1 / 2}\right)$ ions. Unless the relative collision energy is effective in overcoming the endothermicity in the former case, the cross sections for $\mathrm{Xe}^{+}\left({ }^{2} P_{3 / 2}\right)$

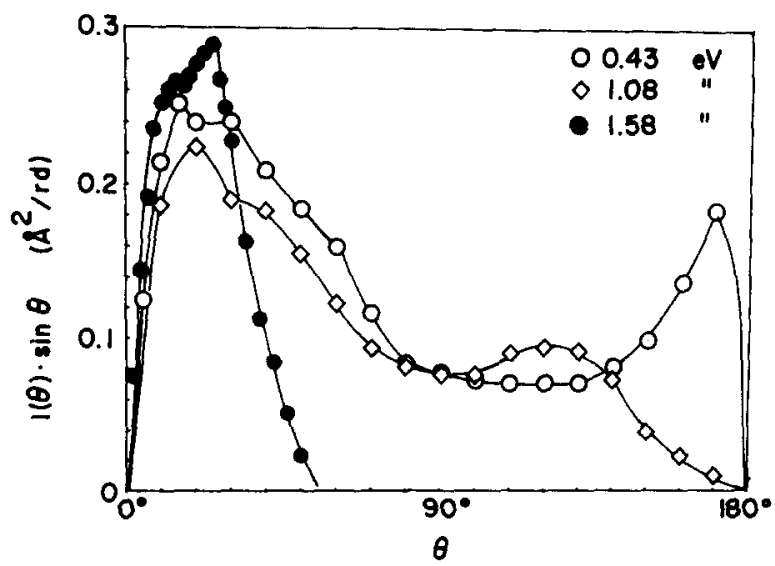

FIG. 10. Polar differential cross sections, $2 \pi I(\theta) \sin \theta$, vs center-of-mass scattering angle $\theta$ for $\mathrm{XeH}^{+}$produced in Reaction (2) at the relative collision energies of $0.43,1.08$, and $1.58 \mathrm{eV}$. These cross sections were obtained by integrating over the product probability distributions shown in Figs. 5-7, using Eq. (8) of the text, and then scaled so that the area under each curve equaled the measured integral reaction cross section at that collision energy, as indicated by Eq. (9).

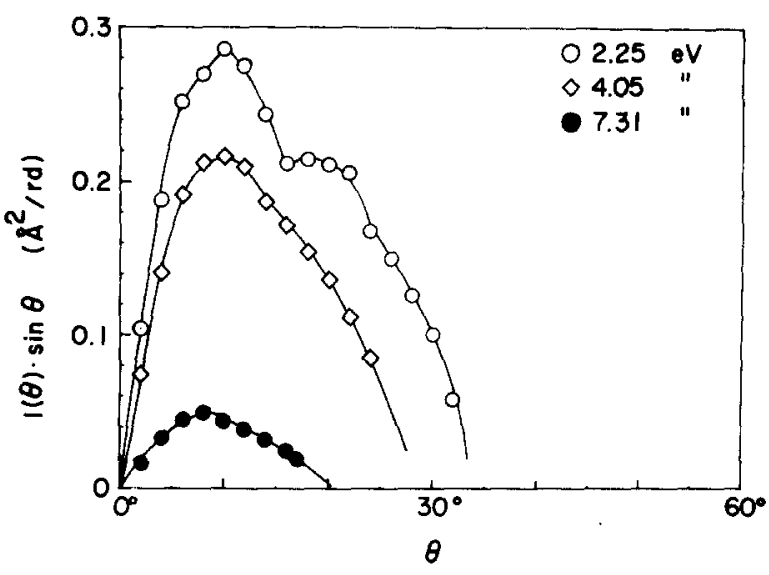

FIG. 11. Polar differential cross sections vs center-of-mass scattering angle for $\mathrm{XeH}^{+}$produced at relative collision energies of $2.25,4.05$, and $7.31 \mathrm{eV}$.

will be much smaller than those for the reaction of the excited $\mathrm{Xe}^{+}$ions. Since the relative population of

$\mathrm{Xe}^{+}\left({ }^{2} P_{1 / 2}\right)$ is $33 \%$ or less for an $\mathrm{Xe}^{+}$beam formed by impact of $30 \mathrm{eV}$ electrons, the actual cross sections for $\mathrm{H}$-atom abstraction by $\mathrm{Xe}^{+}\left({ }^{2} P_{1 / 2}\right)$ are possibly as much as three times larger than the experimental values reported here.

Nevertheless, the cross sections for Reaction (2) are about $\frac{1}{10}$ the value of those for the reaction $\mathrm{Ar}^{+}\left(\mathrm{H}_{2}\right.$, H) $\mathrm{ArH}^{+}$at comparable collision energies. They are, however, similar in magnitude at the higher collision energies to those found previously for $\mathrm{H}$-atom abstraction from $\mathrm{CH}_{4}$ by other simple ions such as $\mathrm{Ar}^{*},{ }^{1,2}$ $\mathrm{Kr}^{*},{ }^{3,4}$ and $\mathrm{N}_{2}^{+} .^{5}$ It has been suggested ${ }^{6}$ that the low yield of $\mathrm{XH}^{+}$from the reactions of these ions with $\mathrm{CH}_{4}$ is a consequence of competition from the very fast exothermic charge transfer processes occurring in these systems. Since $\mathrm{CH}_{4}^{+}$is the major product in reactive $\mathrm{Xe}^{+}-\mathrm{CH}_{4}$ collisions, ${ }^{10}$ the present results are consistent with this suggestion.

Note, however, that the excitation function for Reaction (2) increases monotonically as the collision energy is decreased. In this regard it differs significantly from the excitation functions found for the reactions of other simple ions with methane. In the reactions studied previously, the excitation functions pass through a maximum at a relative collision energy of about $5 \mathrm{eV}$ for reactions of $\mathrm{Ar}^{+}$and $\mathrm{N}_{2}^{+}$and about $3 \mathrm{eV}$ for $\mathrm{Kr}^{+}$; the cross sections decrease at lower energies to such an extent that the $\mathrm{Ar}^{+}$and $\mathrm{N}_{2}^{+}$reactions appear to have translational energy thresholds of about $0.1 \mathrm{eV}$ (c.m.). Because the present study does not include cross sections for Reaction (2) at collision energies below $1.5 \mathrm{eV}$, we cannot rule out the possibility that the excitation function for this reaction would exhibit similar behavior if the collision energy were reduced sufficiently. Nevertheless, the available data give no indication of a maximum in the excitation function for Reaction (2); if one does exist, then it must occur at relative collision energies considerably lower than those found in the other reactions. 


\section{B. Reaction mechanism}

As shown in Figs. 2-4, the $\mathrm{XeH}^{+}$product is strongly scattered in the forward direction (i.e., in the same direction as the incident $\left.\mathrm{Xe}^{+}\right)$at collision energies above $2 \mathrm{eV}$ (c.m.). These velocity vector distributions are very similar in appearance to those reported previous $l y^{1,4}$ for the reactions of $\mathrm{Ar}^{+}$and $\mathrm{Kr}^{+}$with $\mathrm{CH}_{4}$ at comparable collision energies. The observation that the product is asymmetrically distributed about $\pm 90^{\circ}$ in the c. $m$. system is a clear indication that the abstraction of an $\mathrm{H}$ atom from $\mathrm{CH}_{4}$ by $\mathrm{Xe}^{+}$is dominated by a direct mechanism (i.e., an impulsive type of interaction occurring on a time scale comparable to one rotational period, which is on the order of $10^{-13} \mathrm{sec}$ at these energies).

While the $\mathrm{XeH}^{+}$distribution clearly remains forward peaked as the relative collision energy is decreased to $1.58 \mathrm{eV}$ (Fig. 5), there is increased broadening of the distribution and the formation of a low-velocity tail extending toward the center of mass. Again, similar behavior (although somewhat less marked) has been observed at collision energies below $1 \mathrm{eV}$ for the analogous reactions of $\mathrm{Ar}^{+}, \mathrm{Kr}^{+}$, and $\mathrm{N}_{2}^{+}$with $\mathrm{CH}_{4}$. This behavior has been interpreted as an indication of increased interaction between the incident ion and the $\mathrm{CH}_{3}$ at the lower collision energies, with a possible transition from a direct mechanism at higher energies to a long-lived complex mechanism at the very lowest energies. $1,4,5,24,25$

At a relative collision energy of $1.08 \mathrm{eV},($ Fig. 6) the low-velocity tail has extended behind the c.m. into the backward hemisphere, although the peak in the velocity vector distribution still remains considerably forward of the c.m. At $0.43 \mathrm{eV}$ relative collision energy (Fig. 7), the amounts of forward and backward scattering are nearly equal, and the peak of the distribution itself has moved much closer to the c.m.

The contour maps presented here were constructed under the customary assumption of monoenergetic ions colliding with stationary target molecules (i.e., the spread in projectile velocities and the thermal motion of the target molecules have been ignored). In the lowest collision energy experiment $(0.43 \mathrm{eV} \mathrm{c.m}$. ), however, the velocity of the reactant ion is only about four times the most probable speed of the neutral reactant. We have calculated the effects that might be expected from these spreads in projectile and target velocities (see Appendix) in order to determine if they could be responsible for the scattering behavior observed at low collision energy.

Figure 8 shows the calculated distribution functions for the projectile velocity $\left(v_{1}\right)$, target velocity $\left(v_{2}\right)$, relative velocity $(g)$, and the velocity of the center of mass of the system $(V)$, for the experiment at $0.43 \mathrm{eV} \mathrm{c.m}$. The most important consequence of the spreads in reactant velocities is that collisions in a given experiment occur with a relatively broad distribution of relative velocities, $P(g)$. As would be expected from the much greater width of $P\left(v_{2}\right)$ relative to that of $P\left(v_{1}\right)$, the spread of relative velocities (and thus the energy resolution achieved in the present experiments) is limited by the thermal motion of the target molecules rather than by the energy spread of the reactant ion beam.

The principal effect of this spread in collision energies will occur in the measurements of reaction cross sections. The effective cross section $\sigma_{\text {of }}$ measured experimentally at a nominal relative collision energy $T_{0}$ is given by the integral ${ }^{26}$

$$
\sigma_{\text {off }}\left(T_{0}\right)=\int_{0}^{\infty}\left(\frac{T}{T_{0}}\right)^{1 / 2} \sigma(T) f\left(T, T_{0}\right) d T,
$$

where the factor $\left(T / T_{0}\right)^{1 / 2}$ is proportional to the effective path length, $\sigma(T)$ is the actual cross section at relative collision energy $T$, and $f\left(T, T_{0}\right)$ is the distribution function in relative collision energies.

On the other hand, the combination of a heavy projectile and a light target molecule minimizes the target's contribution to the total momentum of the system. Consequently, the distribution of centroids, $P(V)$, is only slightly broader than the distribution in projectile velocities, $P\left(v_{1}\right)$. Since the latter is itself narrow compared to the width of the product velocity distributions (see Figs. 2-7), the kinematic data (product angular and velocity distributions) are relatively unaffected by thermal motion of the target molecules.

Because the reaction cross section is usually a function of relative velocity $g$, the distribution of centroids for those collisions which result in product formation (and thus the product distribution itself) could be shifted in the forward direction for those reactions whose cross sections increase with decrease relative velocity $\left(v_{2}\right.$ parallel to $v_{1}$ ) and shifted in the backward direction for reaction for reactions whose cross sections increase with velocity ( $v_{2}$ antiparallel to $v_{1}$ ). Assuming $\sigma(g)$ $\propto g^{s}$, we have calculated (see Appendix) the distribution of centroids for those collisions resulting in reaction for the three cases $s=-1,0$, and +1 . The results, shown in Fig. 9, indicate that the resulting shift in the distribution of centroids is practically negligible.

In summary, the calculations presented in Figs. 8 and 9 indicate (1) the spread in ion beam velocities and the thermal motion of the target contribute about equally to a broadening of the distribution of centroids; (2) the observed product velocity distribution is considerably broader than the distribution of centroids; and (3) the observed shift of the peak in the product velocity distribution back toward the c.m. at low collision energies is very much greater than could be rationalized by the velocity dependence of the reaction cross section. Thus, the scattering pattern shown in Fig. 7 is most probably a consequence of the reaction dynamics themselves, rather than an experimental artifact caused by the spreads in reactant velocities. This conclusion is supported by the fact that the product distribution shown in Fig. 7 is markedly different from those observed in the earlier studies which showed no backward scattering of the $\mathrm{XH}^{+}$product $\left(\mathrm{X}=\mathrm{Ar}, \mathrm{Kr}\right.$, or $\mathrm{N}_{2}$ ) from methane, even at the lowest collision energies studied.

Therefore, we interpret the shift of the intensity peak toward the c.m. and the increased symmetry of the $\mathrm{XeH}^{*}$ distribution about the $\pm 90^{\circ}$ axis in the c.m. sys - 
tem at $0.43 \mathrm{eV}$ relative collision energy as consequences of a reaction mechanism in which strong coupling and energy exchange between all atoms occurs in many of the collisions at low energy. This raises the possibility that Reaction (2) proceeds, at least in part, via the formation of a $\mathrm{XeCH}_{4}^{+}$collision complex whose lifetime at this energy is comparable to a rotational period.

The direct observation of the $\mathrm{XeCH}_{4}^{+}$ion by Field and Franklin in high-pressure mass spectrometric studies ${ }^{7}$ and by Tiernan and Gill in a tandem mass spectrometric study ${ }^{10}$ indicates the stability of this species under certain conditions. In fact, the appearance potential of the $\mathrm{XeCH}_{4}^{+}$complex was measured ${ }^{7}$ to be $11.7 \mathrm{eV}$, or about $0.5 \mathrm{eV}$ lower than that of $\mathrm{Xe}^{4}\left({ }^{2} P_{3 / 2}\right)$. Presumably the complex ion was produced by an associative attachment reaction between $\mathrm{CH}_{4}$ and an excited Xe atom.

We conclude, therefore, that the product intensity observed behind the c.m. at $1.08 \mathrm{eV}$ (Fig. 6) and the increasing degree of forward-backward symmetry observed at $0.43 \mathrm{eV}$ relative collision energy are probably caused by a transition from a purely direct mechanism at higher energies to one with an increasing contribution from the formation of a long-lived $\mathrm{XeCH}_{4}^{+}$complex at lower energies. The fact that the product distribution at $0.43 \mathrm{eV}$ is slightly forward peaked rather than perfectly symmetric about the $\pm 90^{\circ}$ barycentric axis suggests that the collision complex persists for approximately one rotational period at this energy, rather than for the several rotational periods which would be required to give a totally symmetric distribution.

Additional information on the reaction mechanism can be derived from the product c.m. angular distributions, presented as absolute differential polar cross sections $d \sigma / d \theta$ (Figs . 10 and 11). Two features deserve comment: (1) the near symmetry about $\theta=90^{\circ}$ observed in the $0.43 \mathrm{eV}$ experiment suggests that a complex mechanism predominates at this energy, while the reduced amount of backward scattering found in the $1.08 \mathrm{eV}$ experiment indicates that a transition to a predominantly direct mechanism has occurred by this energy. At higher energies product intensity is entirely restricted to the forward hemisphere, indicating reaction occurs purely in a direct manner. (2) Although the amount of large-angle scattering decreases rapidly with increasing collision energy, the absolute values of the polar differential cross sections for small-angle scattering $\left(\theta<30^{\circ}\right)$ remain essentially constant over the range of collision energies 0.43 to $4.05 \mathrm{eV}$ (c.m.). The observed monotonic decrease in integral reaction cross section with increasing collision energy (Fig. 1) can be attributed exclusively to the rapid decrease in largeangle scattering at higher energies. At relative collision energies below $1 \mathrm{eV}$, this behavior can be attributed to the decreasing probability of complex formation with increasing collision energy. At energies about $1 \mathrm{eV}$, this can no longer be the case since a direct mechanism predominates. Assuming that small-angle scattering in direct reactions arises primarily in grazing encounters resulting from moderately large impact parameter collisions, ${ }^{27}$ we see that such encounters lead to $\mathrm{XeH}^{+}$formation with an efficiency that varies little with collision energy up to $4 \mathrm{eV}$. However, the smaller impact parameter collisions (which would be expected to result in larger-angle scattering) apparently become less effective in producing $\mathrm{XeH}^{+}$as the collision energy is increased. We have observed an increased production of $\mathrm{XeCH}_{3}^{+}$as the collision energy is raised from 1 to $4 \mathrm{eV}$. The fact that the $\mathrm{XeCH}_{3}^{+}$is scattered through c.m. angles greater than $90^{\circ}$ indicates that it is formed predominantly in small impact parameter collisions, so perhaps the decreased ability of such collision to yield $\mathrm{XeH}^{+}$is a consequence of competition from this additional reaction channel.

Information on energy disposal in the reaction can be obtained from the distribution of product relative trans lational energy, $P\left(T^{\prime}\right)$, calculated from Eq. (10). The results are most conveniently expressed in terms of the translational exoergicity $Q$, defined as the difference between the final and initial relative translational energies, $Q=T^{\prime}-T$. This quantity provides a more direct measure of the partitioning of available energy between internal and translational modes of the products than does $T^{\prime}$ itself. Conservation of energy requires that the internal energy of the products, $W^{\prime}$, be given by

$$
W^{\prime}=W-\Delta H-Q .
$$

As discussed in Sec. II, the internal energy of the reactants, $W$, can be taken as zero. Assuming PA (Xe) $=5.09 \mathrm{eV}$ (i.e., the value determined from flowing afterglow measurements of ionic equilibrium), the heat of reaction, $\Delta H$ is +0.86 or $-0.45 \mathrm{eV}$, depending on whether the reactant ion $\mathrm{Xe}^{+}$is in the ${ }^{2} P_{3 / 2}$ or the ${ }^{2} P_{1 / 2}$ state, respectively.

It is possible to assign upper and lower bounds to the translational exoergicity. The maximum value of $Q$ occurs when all the available energy (heat of reaction plus reactant translational and internal energies) appears as product translation. That is,

$$
Q_{\max }=W-\Delta H .
$$

For Reaction (2) the values of $Q_{\max }$ are therefore -0.86 and $+0.45 \mathrm{eV}$ for the ground and the upper state of $\mathrm{Xe}^{+}$, respectively. (The negative value of $Q_{\mathrm{max}}$ for ground state $\mathrm{Xe}^{+}$simply means that $\mathrm{H}$-atom abstraction by $\mathrm{Xe}^{+}\left({ }^{2} P_{3 / 2}\right)$ is only possible if the initial translational energy $T$ exceeds the endothermicity of the reaction, 0.86 eV.)

The minimum value of $Q$ occurs when all of the available energy appears as internal excitation of the reaction products (i.e., when $T^{\prime}=0$ ). Since this would be the case if the reaction proceeded by the formation of a collision complex that decomposed with little or no release of translational energy, this lower limit may be called $Q_{c c}$. It is easily shown that

$$
Q_{c c}=-T \text {. }
$$

Because this implies that the internal energy of the products will increase in a linear manner with increasing collision energy $\left(W^{\prime}=W-\Delta H+T\right)$, there is a lower limit to the value $Q_{c c}$ can assume before product internal excitation has increased to the point where the product will decompose before reaching the detector. If we assume 


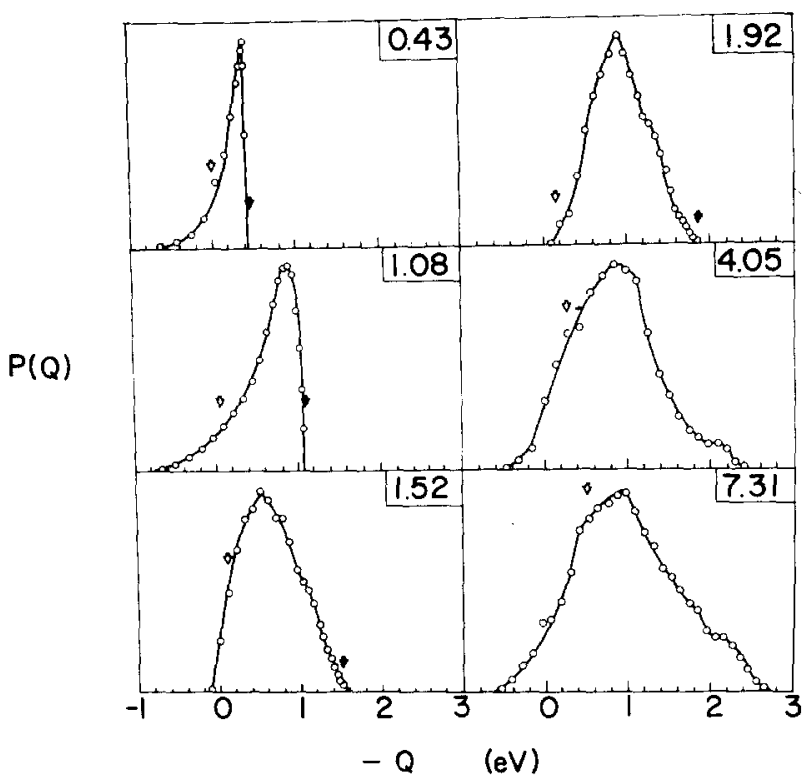

FIG. 12. Product translational exoergicity distributions $P(Q)$ vs $-Q$ for Reaction (2) at six values of the initial relative translational energy. The distributions $P(Q)$ were derived from the complete product probability distributions shown in Figs. 2-7, using Eq. (10) and the definition $Q=T^{\prime}-T$. The solid black arrows in each panel indicate the position of $Q_{c c}$ (i.e., the point at which all available energy appears as internal excitation of the products), while the open arrows indicate the value of $Q$ predicted by the spectator stripping model. The solid lines are smooth interpolations between the experimental points.

that all of the product excitation is contained in the ionic product $\mathrm{XeH}^{+}$, the requirement that this energy not exceed the minimum energy required for dissociation of $\mathrm{XeH}^{+}$

$$
W^{\prime} \leq D_{0}\left[\mathrm{Xe}^{+}\left({ }^{2} P_{3 / 2}\right)-\mathrm{H}\right]=3.62 \mathrm{eV},
$$

yields a pseudominimum for the translational exoergicity:

$$
Q_{\min }=W-\Delta H-D_{0}\left[\mathrm{Xe}^{*}\left({ }^{2} P_{3 / 2}\right)-\mathrm{H}\right] .
$$

Thus, $Q_{\mathrm{mln}}$ is $-4.48 \mathrm{eV}$ for reaction of $\mathrm{Xe}^{+}\left({ }^{2} P_{3 / 2}\right)$ and $-3.17 \mathrm{eV}$ for $\mathrm{Xe}^{+}\left({ }^{2} P_{1 / 2}\right)$. These upper and lower bounds to the translational exoergicity are indicated by the dashed lines in Fig. 13.

The probability $P(Q)$ that products are formed with a given translational exoergicity is plotted in Fig. 12 versus the negative of the translational exoergicity (so that values along the abscissa indicate the net conversion of reactant translational energy to product internal excitation) for experiments performed at six collision energies. The solid black arrow in each panel indicates the position of $Q_{c c}$ (i.e., the point at which all available energy appears as product internal excitation), while the open arrow indicates the translational exoergicity expected on the basis of the spectator stripping model.

The following general features can be discerned from the data shown in Fig. 12. (1) At all collision energies, the overwhelming majority of reactive encounters lead to the net conversion of reactant translational energy to product intermal excitation $(Q<0)$. (2) At collision energies below $2 \mathrm{eV}$, products are formed with internal excitation extending up to the value of the total available energy, $W-\Delta H+T$; at collision energies below $1 \mathrm{eV}$, in fact, the most probable value of $W^{\prime}$ very nearly equals the total available energy. (3) The $P(Q)$ distributions are strongly peaked toward maximum product excitation at low collision energies, but they become more nearly symmetrical at higher energies. (4) The peak in the distribution appears to vary only slowly with collision energy above $T=1.5 \mathrm{eV}$. (5) The spectator stripping model becomes a reasonably accurate predictor of $Q_{m p}$ only at the highest collision energies.

At the lowest collision energy for which a complete distribution was obtained, $0.43 \mathrm{eV}$ (c.m.), the distribution of translational exoergicities is quite narrow and asymmetric, peaking very near the minimum allowed value $\left(Q_{c c}\right)$ of the translational exoergicity and then slowly falling off at more positive values of $Q$. Thus, at this collision energy, nearly all of the available energy appears as internal energy of the reaction products. This behavior, which is what one would expect if reaction proceeded via the formation of a long-lived collision complex, also occurs at $T=1.08 \mathrm{eV}$, but not at the higher collision energies. This behavior appears to be a consequence of the transition from a complex mechanism at low collision energies ( $T<1 \mathrm{eV}$ ) to a direct mechanism at higher energies.

The dependence of the reaction mechanism upon collision energy is summarized in Fig. 13, where the most probable value of the translational exoergicity, $Q_{m p}$, is plotted versus $T$. At collision energies lower than about $0.5 \mathrm{eV}$, the most probable value of $Q$ is seen to be $Q_{\mathrm{cc}}$, indicating that $\mathrm{XeH}^{+}$is formed in a perfectly inelastic collision (as, for example, by the decomposition of a "sticky" collision complex with the release of little or no translational energy). Above $0.5 \mathrm{eV}$, however, $Q_{\mathrm{mp}}$

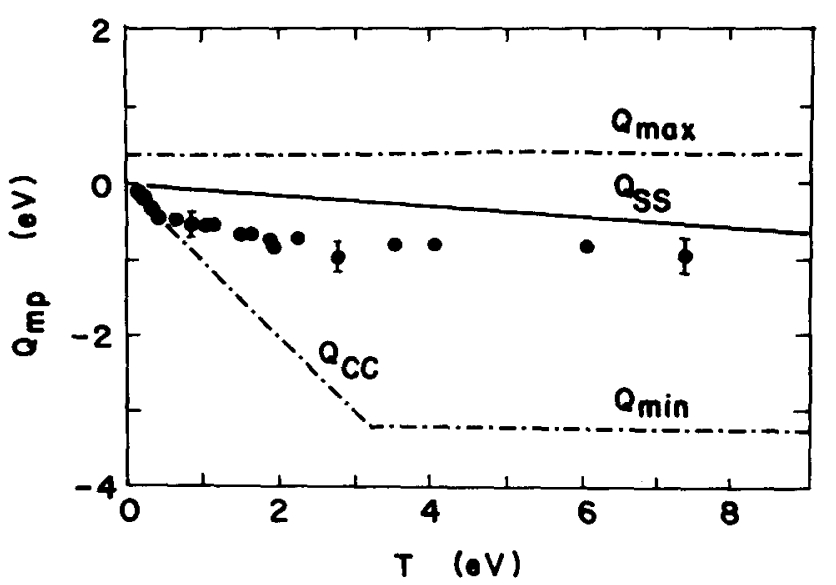

FIG. 13. Most probable translational exoergicity $Q_{\operatorname{mg}}$ vs initial translational energy $T$ for Reaction (2). The lines labeled $Q_{\max }$ and $Q_{\min }$ represent $0 \%$ and $100 \%$ conversion of available energy to product internal excitation, respectively, assuming the reactant ion is $\mathrm{Xe}^{+}\left({ }^{2} P_{1 / 2}\right)$. The prediction of the spectator stripping model is shown by the line labeled $Q_{\mathrm{ss}}$. Estimated experimental uncertainties in $Q_{m p}$ are indicated by error bars for several selected points. 
decreases (i.e., $W_{m p}^{\prime}$ increases) only slowly with increasing collision energy, indicating that only a small fraction $(<10 \%)$ of this additional collision energy is partitioned into product excitation. In fact, the rate of this decrease in $Q_{\text {mp }}^{\prime}$ is approximately that expected from the SSM model, although the absolute value of $Q_{\mathrm{mp}}^{\prime}$ (and therefore $T_{m p}^{\prime}$ ) rather consistently remains $0.4-0.5 \mathrm{eV}$ less than predicted by the SSM. Although such negative deviations from the SSM were also observed for the reactions of $\mathrm{Ar}^{+}, \mathrm{Kr}^{*}$, and $\mathrm{N}_{2}^{+}$with $\mathrm{CH}_{4}$, the magnitude of this deviation observed in the present study $(0.5 \mathrm{eV})$ is considerably greater than those found $(0.1-0.2 \mathrm{eV})$ in the earlier studies.

We have discussed ${ }^{1}$ in some detail the possibility that the negative deviations from the SSM observed in these reactions are caused by an attractive potential between the reaction products which produces a basin in the potential energy hypersurface from which the products can escape only with difficulty, particularly at the lowest collision energies. Direct reaction is possible only if the collision energy is high enough and the geometry of the collision such that the newly formed products retain sufficient momentum to escape from the potential basin and separate completely. The data of Fig. 13 indicate that this critical collision energy (the stripping threshold) above which direct reaction is possible in the $\mathrm{Xe}^{*}$ $+\mathrm{CH}_{4}$ reaction is approximately $0.5 \mathrm{eV}$. At lower collision energies the colliding species become trapped in the potential basin to form an $\mathrm{XeCH}_{4}^{+}$complex, several oscillations of which may be required before the $\mathrm{XeH}^{+}$ can give the $\mathrm{CH}_{3}$ a clout sufficient to complete separation.

In the reactions of $\mathrm{Ar}^{*}, \mathrm{Kr}^{*}$, and $\mathrm{N}_{2}^{+}$with $\mathrm{CH}_{4}$ a similar intermediate complex may have formed and then decomposed via one or more fast, exothermic charge transfer channels before sufficient internal energy transfer occurred for the escape of the $\mathrm{XH}^{+}$product. If we assume that the $\mathrm{Xe}^{+}\left({ }^{2} P_{1 / 2}\right)$ ion is the precursor of the $\mathrm{XeH}^{*}$ observed in the present study (because reaction of the ground state $\mathrm{Xe}^{+}$ion is endothermic), charge transfer to $\mathrm{CH}_{4}$ is also exothermic and thus a competing pathway for the decomposition of the collision complex in this system. However, the observation that the cross sections for $\mathrm{XeH}^{+}$formation do not decrease at low collision energies (as the cross sections for $\mathrm{XH}^{+}$formation did in the case where $\mathrm{X}=\mathrm{Ar}, \mathrm{Kr}$, and $\mathrm{N}_{2}$ ) indicates that competition from charge transfer inhibits $\mathrm{H}$-atom $\mathrm{ab}-$ straction less seriously in the $\mathrm{Xe}^{+}$reaction than in the others. A possible explanation for this difference can be provided by statistical theory, which predicts that decomposition of a collision complex will favor the most exothermic channel because of its greater density of states (see, for example, Ref. 27, p. 223). Charge transfer is considerably more exothermic than $\mathrm{XH}^{+}$formation for those system $\left(X=A r, K r\right.$, and $\left.N_{2}\right)$ which show a decrease in cross section for $\mathrm{XH}^{+}$formation at low collision energies. For the $\mathrm{Xe}^{+}$case, however, the two channels have comparable exothermicities; consequently, the statistical bias favoring charge transfer over abstraction in the decomposition of any collision complex formed at low collision energy would be considerably less in the $\mathrm{Xe}^{+}$reaction than in the others.

\section{SUMMARY AND CONCLUSIONS}

Integral cross sections and product velocity vector distributions have been obtained for the reaction $\mathrm{Xe}^{+}$ $+\mathrm{CH}_{4}-\mathrm{XeH}^{*}+\mathrm{CH}_{3}$ as a function of reactant translational energy. Available thermochemical data indicates that this reaction is endothermic by $0.86 \mathrm{eV}$ for ground state $\mathrm{Xe}^{*}$ ions but exothermic by $0.45 \mathrm{eV}$ for the upper level of the $\mathrm{Xe}^{+}$doublet. It is likely, therefore, that the data reported here principally reflect the reaction of $\mathrm{Xe}^{+}\left({ }^{2} P_{1 / 2}\right)$ with $\mathrm{CH}_{4}$.

At relative collision energies above $\approx 1 \mathrm{eV}$, the abstraction reaction is dominated by a direct mechanism. The spectator stripping mechanism provides only a poor approximation to the reaction dynamics, the most probable product translational energy consistently being about $0.5 \mathrm{eV}$ less than that predicted by the spectator stripping model. At relative collision energies below $\approx 1 \mathrm{eV}$, there appears to be a transition in the reaction mechanism to one involving the formation of a long-lived $\mathrm{XeCH}_{4}^{+}$collision complex.

Reaction cross sections for $\mathrm{XeH}^{+}$formation are small (0.1 to $1.0 \AA^{2}$ ), possibly as a consequence of competition from charge transfer to form $\mathrm{CH}_{4}^{+}$. However, the $\mathrm{XeH}^{+}$ cross sections do not exhibit the sharp decrease at low collision energies observed for the corresponding reactions of $\mathrm{Ar}^{+}, \mathrm{Kr}^{+}$, and $\mathrm{N}_{2}^{+}$with $\mathrm{CH}_{4}$. We attribute this to the differences in the ionization potentials of the various reactant ions. Because charge transfer from $\mathrm{Xe}^{+}$to $\mathrm{CH}_{4}$ is only slightly more exothermic than $\mathrm{XeH}^{+}$formation, there is not the strong statistical factor favoring charge transfer in the decomposition of any $\mathrm{XCH}_{4}^{+} \mathrm{col}-$ lision complex that there is in the other three cases.

\section{ACKNOWLEDGMENTS}

Acknowledgment is made to the Kansas General Research Fund for support of this research. We would like to thank Professor Robert Sampson and the Kansas Geological Survey for providing the computer programs used to construct the intensity contour diagrams.

\section{APPENDIX}

If $v_{1}$ and $v_{2}$ are the laboratory velocities of the ionic and neutral reactants, respectively, the relative velocity $g$ of the two particles is

$$
g=v_{1}-v_{2} \text {. }
$$

If $P_{1}\left(v_{1}\right)$ and $P_{2}\left(v_{2}\right)$ represent the distributions in ionic and neutral reactant velocities, the distribution in $g$ is found by convoluting $P_{1}\left(v_{1}\right)$ with $P_{2}\left(v_{2}\right)$, subject to the condition that $v_{2}=v_{1}-g$ :

$$
P(g)=\int_{-\infty}^{\infty} P_{1}\left(v_{1}\right) \cdot P_{2}\left(v_{1}-g\right) d v_{1}
$$

In the approximate one-dimensional treatment employed here, the distribution of target velocities along the $0^{\circ}$ direction is given by the one-dimensional Maxwellian distribution function,

$$
P_{2}\left(v_{2}\right) \propto \exp \left[-\left(v_{2} / \alpha\right)^{2}\right]
$$


where $\alpha=\left(2 k T / m_{2}\right)^{1 / 2}, k$ being the Boltzmann constant and $T$ the target gas temperature.

We have found that, for our instrument, the distribution of reactant ion velocities in the $0^{\circ}$ direction is well approximated by a Gaussian distribution centered around the most probable ionic velocity, $\bar{v}_{1}$ :

$$
P_{1}\left(v_{1}\right) \propto \exp \left[-\left(\frac{v_{1}-\bar{v}_{1}}{\sigma_{1}}\right)^{2}\right]
$$

where $\sigma_{1}$ is related to the measured full width of the ionic velocity distribution at half its maximum (FWHM) by $\sigma_{1}$ $=$ FWHM $/ 2 \sqrt{\ln 2}$.

Substitution of Eqs. (A3) and (A4) into (A2), followed by integration of the resulting expression, yields the result

$$
P(g) \propto \exp \left[-\left(\frac{g-\bar{v}_{1}}{\sigma_{g}}\right)^{2}\right],
$$

where $\sigma_{g}^{2}=\alpha^{2}+\sigma_{1}^{2}$.

The velocity $V$ of the center of mass of the two particles is related to their velocities by the expression

$$
V=\gamma v_{1}+(1-\gamma) v_{2}
$$

where $\gamma=m_{1} /\left(m_{1}+m_{2}\right), m_{1}$ and $m_{2}$ being the masses of the ionic and the neutral reactants, respectively. The distribution in $V$ is found by convoluting $P_{1}\left(v_{1}\right)$ with $P_{2}\left(v_{2}\right)$, subject to the condition that $v_{2}=\left(V-\gamma v_{1}\right) /(1-\gamma)$ :

$$
P(V)=\int_{-\infty}^{\infty} P_{1}\left(v_{1}\right) \cdot P_{2}\left(\frac{V-\gamma v_{1}}{1-\gamma}\right) d v_{1} \text {. }
$$

Substitution of Eqs. (A3) and (A4) into (A7), followed by integration of the resulting expression, yields

$$
P(V) \propto \exp \left[-\left(\frac{V-\bar{V}}{\sigma_{\mathrm{cm}}}\right)^{2}\right]
$$

where $\bar{V}$, the nominal velocity of the c.m. is given by $\bar{V}=\gamma \bar{v}_{1}$ and $\sigma_{\mathrm{cm}}^{2}=\gamma^{2} \sigma_{1}^{2}+(1-\gamma)^{2} \alpha^{2}$. The distribution functions $P_{1}\left(v_{1}\right), P_{2}\left(v_{2}\right), P(g)$, and $P(V)$ are presented graphically in Fig. 8.

Since a given c.m. velocity $V$ can result from any combination of velocities $v_{1}$ and $v_{2}$ that satisfy Eq. (A6), collisions will occur over a range of relative velocities $g$ for a given value of $V$. The relative probability that collisions with a c.m. velocity $V$ lead to reaction is given by

$P_{r \times n}(V)=\int_{-\infty}^{\infty}\left(\frac{g}{\bar{v}_{1}}\right) \sigma(g) \cdot P_{1}\left(v_{1}\right) \cdot P_{2}\left(\frac{V-\gamma v_{1}}{1-\gamma}\right) d v_{1}$.

For a given value of $V, g=\left(v_{1}-V\right) /(1-\gamma)$. Assuming the dependence of the reaction cross section on relative velocity is given by $\sigma(g) \propto g^{s}, \mathrm{Eq}$. (A9) can be written as

$$
P_{r \times n}(V) \propto \int_{-\infty}^{\infty}\left|v_{1}-V\right|^{* 1} P_{1}\left(v_{1}\right) \cdot P_{2}\left(\frac{V-\gamma v_{1}}{1-\gamma}\right) d v_{1} .
$$

After substitution of the appropriate expressions for $P_{1}$ and $P_{2}, \mathrm{Eq} .(\mathrm{A} 10)$ can be written in the form

$$
P_{r \times n}(V) \propto \exp \left(-\frac{\left(V-\vec{V}^{2}\right.}{\sigma_{\mathrm{cm}}^{2}}\right) I(V)=P(V) \cdot I(V),
$$

where the integral $I(V)$, which is the relative probability for reaction at a given value of $V$, is
TABLE II. Values of parameters for velocity distributions in the reaction $\mathrm{Xe}^{+}$with $\mathrm{CH}_{4}$ at a relative collision energy of $0.43 \mathrm{eV}$.

\begin{tabular}{cc}
\hline \hline Parameter & Value $^{\mathrm{a}}$ \\
\hline$\gamma$ & 0.892 \\
$\bar{v}_{1}$ & 25.74 \\
$\bar{V}$ & 22.96 \\
$\alpha$ & 6.10 \\
$\sigma_{1}$ & 0.711 \\
$\sigma_{\mathrm{cm}}$ & 0.914 \\
$\sigma_{\mathrm{s}}$ & 6.14 \\
\hline \hline
\end{tabular}

${ }^{2}$ Velocities are expressed in units of $10^{4} \mathrm{~cm} / \mathrm{sec}$.

$$
I(V)=\int_{-\infty}^{\infty}\left|v_{1}-V\right|^{s+1} \exp \left[-\left(\frac{a v_{1}-b}{c}\right)^{2}\right] d v_{1},
$$

where

$$
\begin{aligned}
& a=\left[\gamma^{2} \sigma_{1}^{2}+(1-\gamma)^{2} \sigma^{2}\right]^{1 / 2}=\sigma_{\mathrm{cm}}, \\
& b=\frac{\alpha^{2}(1-\gamma)^{2} \bar{v}_{1}+\sigma_{1}^{2} \gamma V}{c}, \\
& c=(1-\gamma) \alpha \sigma_{1} .
\end{aligned}
$$

Making the substitution $X=\left(a v_{1}-b\right) / c, d=(b-a V) / c$, Eq. (A11) becomes

$$
I(V) \propto \int_{-\infty}^{\infty}|X+d|^{s+1} e^{-x^{2}} d X .
$$

To evaluate the effect of path length and reaction cross section on the distribution of centroids for reactive collisions, the integral $I(V)$ has been evaluated for three types of reaction cross section

Case 1. Cross sections which decrease with relative velocity as $g^{-1}$ (i.e., follow the Langevin equation). With $s=-1$, Eq. (A16) becomes

$$
I(V) \propto \int_{-\infty}^{\infty} e^{-X^{2}} d X=\sqrt{\pi} .
$$

Case 2. Cross sections which are independent of relative velocity (i.e., hard-sphere collisions). With $s=0$, Eq. (A16) becomes

$$
\begin{aligned}
I(V) & \propto \int_{-\infty}^{\infty}|X+d| e^{-x^{2}} d X \\
& =\sqrt{\pi} d \operatorname{erf}(d)+e^{-d^{2}} \\
& \simeq \sqrt{\pi} d \text { when } d>3 .
\end{aligned}
$$

Case 3. Cross sections which increase linearly with relative velocity. With $s=+1$, Eq. (A16) becomes

$$
\begin{aligned}
I(V) & =\int_{-\infty}^{\infty}(X+d)^{2} e^{-x^{2}} d X \\
& =\sqrt{\pi}\left(d^{2}+\frac{1}{2}\right) .
\end{aligned}
$$

From the definition of $d$, we can write

$$
d=\frac{\alpha^{2}(1-\gamma)\left(\bar{v}_{1}-V\right)+\gamma \sigma_{1}^{2} V}{\alpha \sigma_{1} \sigma_{\mathrm{cm}}} .
$$


Using the values of the parameters listed in Table II, Eq. (A20) becomes $d=26.19-0.9 V$, where $V$ is expressed in units of $10^{4} \mathrm{~cm} / \mathrm{sec}$. The expressions obtained for $I(V)$ [Eqs. (A17)-(A19)] have been used to calculate $P_{r \times n}(V)$ for the three cases, $s=+1,0,-1$. The results are shown graphically in Fig. 9.

1J. R. Wyatt, L. W. Strattan, S. C. Snyder, and P. M. Hierl, J. Chem. Phys. 62, 2555 (1975).

${ }^{2}$ J. R. Wyatt, L. W. Strattan, S. Chivalak, and P. M. Hierl, J. Chem. Phys. 63, 4582 (1975).

${ }^{3}$ J. R. Wyatt, L. W. Strattan, and P. M. Hierl, J. Chem. Phys. 63, 5044 (1975).

${ }^{4}$ J. R. Wyatt, L. W. Strattan, and P. M. Hier1, J. Phys. Chem. 80, 2911 (1976).

${ }^{5}$ J. R. Wyatt, L. W. Strattan, S. C. Snyder, and P. M. Hierl, J. Chem. Phys. 64, 3757 (1976).

${ }^{6}$ V. L. Talrose and G. V. Karachevtsev, Adv. Mass Spectrom. 3, 211 (1966); A. J. Masson, K. Birkinshaw, and M. J. Henchman, J. Chem. Phys. 50, 4112 (1969).

${ }^{7}$ J. L. Franklin and F. H. Field, J. Am. Chem. Soc. 83, 4509 (1961).

${ }^{8}$ D. Holtz and J. L. Beauchamp, Science 173, 1237 (1971). ${ }^{9} \mathrm{~J}$. H. Futrell and R. P. Clow, J. Chem. Phys. 50, 5041 (1969).

${ }^{10}$ T. O. Tiernan and P. S. Gill, J. Chem. Phys. 50, 5042 (1969).
${ }^{11} \mathrm{~A}$. J. Masson, Ph. D. thesis (Brandeis University, 1970).

${ }^{12} \mathrm{Z}$. Herman, J. D. Kerstetter, T. L. Rose, and R. Wolfgang, Rev. Sci. Instrum. 40, 538 (1969).

${ }^{13} \mathrm{C}$. F. Giese, Rev. Sci. Instrum. 30, 260 (1959).

${ }^{14} \mathrm{P}$. M. Hierl, L. W. Strattan, and J. R. Wyatt, Int. J. Mass Spectrom. Ion Phys. 10, 385 (1973).

${ }^{15}$ H. D. Hagstrum, Phys. Rev. 104, 309 (1956).

${ }^{16}$ Natl. Bur. Stand. Ref. Data Ser. 26 (1969).

${ }^{17}$ W. A. Chupka, J. Chem. Phys. 48, 2337 (1968).

${ }^{18} \mathrm{~F}$. C. Fehsenfeld, W. Lindinger, H. I. Schiff, R. S. Hemsworth, and D. K. Bohme, J. Chem. Phys. 64, 4887 (1976).

${ }^{19} \mathrm{~K}$. E. McCulloh, presented at the Twenty-seventh Annual Conference on Mass Spectrometry and Allied Topics, Seattle, Washington, June 3-8, 1979.

${ }^{20} \mathrm{C}$. Kubach, V. Sidis, and J. Durup, J. Phys. B 8, 1129 (1975).

${ }^{21}$ H. P. Weise, H. U. Mittmann, A. Ding, and A. Henglein, Z. Naturforsch. A 26, 1122 (1971).

${ }^{22}$ G. Herzberg, Spectra of Diatomic Molecules (Van Nostrand, Princeton, New Jersey, 1950), p. 540.

${ }^{23}$ R. Wolfgang and R. J. Cross, J. Phys. Chem. 73, 743 (1969); P. M. Hierl, Z. Herman, and R. Wolfgang, J. Chem. Phys. 53, 660 (1970).

${ }^{24} \mathrm{~A}$. Ding, A. Henglein, D. Hyatt, and K. Lacmann, Z. Naturforsch. A 23, 2084 (1968).

${ }^{25}$ A. Henglein, J. Chem. Phys. 53, 458 (1970).

${ }^{26} \mathrm{P}$. J. Chantry, J. Chem. Phys. 55, 2746 (1971).

${ }^{27} \mathrm{See}$, for example, R. B. Bernstein and R. D. Levine, Molecular Reaction Dynamics (Oxford University, New York, 1974), pp. $76-77$. 